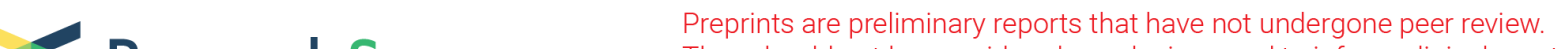 Research Square They should not be considered conclusive, used to inform clinical practice, or referenced by the media as validated information.
}

\section{Marine Furanocembranoids-Inspired Macrocycles Enabled by Pd-catalyzed Unactivated C(sp3)-H Insertion Reaction of Donor/Donor Carbenes}

Jiping Hao

Shanghai Institute of Materia Medica

\section{Xueying Guo}

The Hong Kong University of Science and Technology https://orcid.org/0000-0003-2056-8827

\section{Shijun He}

Shanghai Institute of Materia Medica

\section{Zhongliang Xu}

Shanghai Institute of Materia Medica

\section{Lu Chen}

Shanghai Institute of Materia Medica

\section{Zhongyu Li}

Shanghai Institute of Materia Medica

\section{Bichao Song}

Shanghai Institute of Materia Medica

\section{Jianping Zuo}

Shanghai Institute of Materia Medica

\section{Zhenyang Lin}

The Hong Kong University of Science \& Technology https://orcid.org/0000-0003-4104-8767

Weibo Yang ( $\square$ yweibo@simm.ac.cn)

Shanghai Institute of Materia Medica https://orcid.org/0000-0003-1633-7655

\section{Article}

Keywords: Biomimetic Modularization, Function Oriented Synthesis, Diazo Compounds, Allenyl Ketones Carbene Precursors, Dexamethosone

Posted Date: September 25th, 2020

DOI: https://doi.org/10.21203/rs.3.rs-58031/v1

License: (c) (1) This work is licensed under a Creative Commons Attribution 4.0 International License. Read Full License 
Version of Record: A version of this preprint was published at Nature Communications on February 26th, 2021. See the published version at https://doi.org/10.1038/s41467-021-21484-x. 


\section{Abstract}

Biomimetic modularization and function-oriented synthesis of structurally diversified natural product-like macrocycles in a step-economical fashion is highly desirable. Inspired by marine furanocembranoids, herein, we unprecedentedly synthesized diverse alkenes substituted furan-embedded macrolactams via a modular biomimetic assembly strategy. The success of this assembly is the development of crucial Pdcatalyzed carbene coupling between ene-yne-ketones as donor/donor carbene precursors and unactivated $\mathrm{Csp} 3-\mathrm{H}$ bonds which represents a great challenge in organic synthesis. Notably, this method not only obviates the use of unstable, explosive, and toxic diazo compounds, but also can be amenable to allenyl ketones carbene precursors. DFT calculations demonstrated that a 1,4-Pd shift could be involved in the mechanism. Moreover, the collected furanocembranoids-like macrolactams showed significant anti-inflammatory activities against TNF- $\alpha, \mathrm{IL}-6$, and IL-1 $\beta$ and the low cytotoxicity is comparable to Dexamethasone.

\section{Introduction}

Many natural macrocyclic small-molecules have evolved to interfere with protein-protein interactions, and often have been harnessed as probes for target validation and starting points for lead compounds for drugs discovery. ${ }^{1-7}$ For example, marine cembranoids or furanobutenolide-based cembranoids exhibit a wide range of biological activities, e.g. antitumoral, antimicrobial and anti-inflammatory (Fig. 1A). A deeper examination into their scaffolds reflects that alkene substituted furan is of crucial importance for the biological activities. Despite these valuable function, gene expression limitations of soft corals and difficulty of resupply could hamper the sustainability of them. Therefore, the development of new strategies and methods to expeditiously access and enrich diverse natural furanocembranoids-like chemical space is highly desirable. ${ }^{8-14}$ Inspired by natural products or privileged scaffold ${ }^{15}$ and our interest in developing new coupling reactions, ${ }^{16-19}$ we set out to create polysubstituted alkene furanembedded macrolactams via a short and modular biomimetic strategy, which simply utilizes either the fundamental building blocks from living organism's endogenous ligands or mimics, such as amino acids or unnatural amino acids. ${ }^{20-21} \mathrm{~A}$ retrosynthetic analysis indicated that a successive and concise $\mathrm{Csp}{ }^{3}-\mathrm{H}$ carbene coupling, and amidation could assemble these readily available building blocks, such as aryl bromides, natural or unnatural amino acids and enynones, faithfully into the target molecules (Fig. 1B).

Over the past decade, transition-metal (e.g., Pd, Rh, Ir)-catalyzed carbene cross-coupling ${ }^{22}$ has emerged as a powerful tool for organic chemists to construct polysubstituted alkenes that might be difficult to synthesize by other methods. Concomitant with the rapid growth of this field is the combination of $\mathrm{C}-\mathrm{H}$ activation ${ }^{23-25}$ and carbene cross-coupling due to the remarkable advantage in atom- and step-economy. In this context, the functionalizations of $\mathrm{Csp}^{2}-\mathrm{H}$ substrates by using diazo esters as acceptor carbene precursors are highly advanced. ${ }^{26-29}$ While a few carbene cross-couplings of $\mathrm{Csp}^{3}-\mathrm{H}$ substrates with diazo esters have been developed, they are mainly limited to the activated positions (benzylic, allylic, a-to heteratoms).${ }^{30-35}$ Significant progress has been made in carbene cross-couplings of unactivated $\mathrm{Csp}^{3}-\mathrm{H}$ 
bonds from Davies ${ }^{36-41}$ and Martin. ${ }^{42}$ Notably, all of these reported protocols exclusively employed the donor-acceptor metal carbenoid generated from diazo esters. To the best of our knowledge, the carbene cross-couplings of unactivated $\mathrm{Csp}^{3}-\mathrm{H}$ bonds with non-diazo donor/donor carbene precursors have never been documented, although they are more environmentally friendly and could hold great promise for the exploration of chemical space. Expanding this carbene chemistry to unactivated $\mathrm{Csp}^{3}-\mathrm{H}$ bonds with non-diazo donor/donor carbene is elusive for several reasons. First, metal-mediated cleavage of unactivated $\mathrm{Csp}^{3}-\mathrm{H}$ bonds is often significantly slower than $\mathrm{Csp}^{2}-\mathrm{H}$ bonds. Second, the electrophilic capacity of donor/donor carbene is relatively weak compared to the acceptor carbene. Finally, the key C$\mathrm{C}$ bond-forming event requires metal carbenoid intermediate. Such intermediates are frequently plagued by competing oxidation and dimerization. ${ }^{41}$

Herein, we report the first $\mathrm{Pd}$-catalyzed intermolecular unactivated $\mathrm{Csp}^{3}-\mathrm{H}$ bond insertion reactions by choosing ene-yne-ketones and allenyl ketones as donor/donor carbene precursors. ${ }^{43-51}$ Accordingly, these reactions are distinguished by their high stereoselectivity and wide substrate scope including several drug derivatives. DFT mechanistic studies reveal that a 1,4-Pd shift could be involved. ${ }^{52-61}$ The unique features of these alkenes substituted furans are illustrated as novel building blocks for the construction of anti-inflammatory ${ }^{62}$ macrocyclic targets.

\section{Results}

Stimulated by the challenges of our synthetic target macrolactams, we first examined the module assembly by optimizing unactivated $\mathrm{Csp}^{3}-\mathrm{H}$ olefination. 1-Bromo-2-(tert-butyl)benzene and 3-(4,4dimethylpent-2-yn-1-ylidene)pentane-2,4-dione were selected as the model substrates and a number of reaction parameters such as base, ligand, Pd catalyst, and solvents were screened. After considerable experimentation, we were pleased to discover that a simple cocktail containing $[\mathrm{PdCl}(\text { allyl })]_{2}(5 \mathrm{~mol} \%)$, ${ }^{\mathrm{t} B u X p h o s}(30 \mathrm{~mol} \%)$, and $\mathrm{NaOAc}$ in DMF at $100^{\circ} \mathrm{C}$ in 1,4-dioxane established the reaction conditions, affording compound 3 aa in $76 \%$ isolated yield with high stereoselectivity after $4 \mathrm{~h}$ (Table 1 , entry 1 ). This $\mathrm{Csp}^{3}-\mathrm{H}$ olefination is distinctive from Martin and co-workers' recent work, ${ }^{42}$ in which they described an interesting Pd-catalyzed [4+1] cycloaddition of diazo esters. A series of control experiments were also conducted to validate the role of each parameter. Not surprisingly, the examined parameters were all essential for this transformation. The use of either DIPEA or KOAc did not further improve the yield of the desired product 3aa (Table 1, entries 2 and 3). Notably, the ligand appears to be important, as replacing tBuXphos with Xphos or Brettphos provided 3aa in a much lower yield and no reaction occurred in the absence of ligand (Table 1, entries 6, 7, and 8). In addition, a diminished yield was observed when $\mathrm{Pd}(\mathrm{MeCN})_{4}(\mathrm{OTf})_{2}$ or $\mathrm{Pd}(\mathrm{OAc})_{2}$ was employed (Table 1, entries 9 and 10). The influence of the solvents was also investigated. While similar efficiency was obtained using DMA, only traces of product were obtained in THF and no detectable amount of 3aa could be found in acetonitrile (Table 1, entries 13 and 14). 
After determining the optimal reaction conditions, we turned our attention to evaluate the scope of this $\mathrm{Pd}$-catalyzed intermolecular unactivated $\mathrm{Csp}^{3}-\mathrm{H}$ bond insertion reaction with ene-yne-ketones as donor/donor carbene precursors. As shown in Table 2, our $\mathrm{Csp}^{3}-\mathrm{H}$ carbene olefination method turned out to be widely applicable regardless of the electronic variations at the para and meta positions on the aromatic ring of the aryl bromides (3aa-3ea). Likewise, the naphthyl bromide employed for the synthesis of 3fa served well as a partner in the reaction. Gratifyingly, functional groups on the tertiary alkyls including cyano and ester are compatible (3ga-3ha), although aryl, secondary, and primary alkyls are not reactive probably due to steric hindrance or $\beta$-H elimination. ${ }^{63-66}$ Particularly interesting was the observation that the aryl bromide substrate substituted with free amine did not interfere, providing 3ja in a good yield without traces of the $\mathrm{N}-\mathrm{H}$ bond carbene insertion product being observed. Remarkably, the ene-yne-ketones containing ketone, ester, and heterocyclic ring can be successfully transformed into corresponding products (3ab-3ad) in good to excellent yields (77-91\%).

To evaluate the generality of the protocol, alternatively, we investigated this $\mathrm{Csp}^{3}-\mathrm{H}$ carbene olefination process using allenyl ketones as donor/donor carbene precursors. ${ }^{47}$ As illustrated in Table 3, a wide range of allenyl ketones with electron-donating or -withdrawing substituents were well tolerated and a series of alkenes derivatives substituted with dihydrofurans were obtained. Generally, reactions of allenyl ketones with electron-donating substituents attached to a phenyl ring proceeded in higher yields than those having electro-withdrawing groups (5ab, 5ac, 5ad, 5ah). Moreover, the relative configuration of $5 \mathrm{ab}$ was unambiguously assigned by the X-ray crystal structure analysis. Particularly, substrates bearing furanyl and thienyl functional groups were also amenable to the standard conditions, which provided the pharmaceutical bis-heterocyclic compounds in decent yields with excellent stereoselectivities.

The identification of lead compounds greatly benefits from fragment-based drug design and the ability to directly modify the privileged scaffolds. Therefore, to highlight the potential application of these $\mathrm{Csp}^{3}-\mathrm{H}$ bond carbene coupling reactions in medicinal chemistry, late-stage cyclization/olefination of complex and bioactive molecules was subjected to our established protocol. Remarkably, the alkenes substituted with furans and dihydrofurans products derived from Repaglinide, Isoxepac, Mycophenolic acid, Adapalene and Dehydrocholic acid were synthesized in moderate to excellent yields (Table 4). For example, Repaglinide, an antidiabetic drug used to control blood sugar in type 2 diabetes mellitus, had also been installed with 1-bromo-2-(tert-butyl)benzene and subjected into this protocol, gave access to the product $3 \mathbf{k a}$ in an excellent $92 \%$ yield. Notably, starting from Isoxepac, a non-steroidal anti-inflammatory drug with analgesic activity, which was successfully converted to new furan or dihydrofuran-containing Isoxepac (3la, 5lc) in $84 \%$ and $74 \%$ yield, respectively.

Once the crucial connection of the aryl bromides and enynone building blocks was successfully established, we next selected different natural or unnatural amino acids and attempted to assemble them 
to the macrolactams via a short and modular biomimetic strategy. With 6-8 steps, eight novel polysubstituted alkene-embedded macrolactams $(\mathbf{6 a}-\mathbf{6 h})$ were efficiently assembled (Scheme 1$)$. To explore whether these alkene-embedded macrolactams could successfully exhibit pharmacologically relevant features, the macrolactams $6 \mathbf{a}-6 \mathrm{~h}$ were investigated for the inhibitory effects on inflammatory mediators by LPS-induced inflammatory responses in RAW 246.7 macrophages. The results showed that $\mathbf{6 g}$ exhibited prominent inhibitory effects on the production of TNF-a, IL-6, and IL-1 $\beta$ with IC50 values of $0.45,1.59$, and $0.59 \mu \mathrm{M}$, respectively. It should be noted that these pro-inflammatory cytokines are critically involved in the process of inflammation, immunity, cell survival and apoptosis, and metabolic diseases. ${ }^{67-69}$ Both $\mathbf{6 g}$ and $\mathbf{6 h}$ were approximately 10 times more potent in the inhibitory activity on IL-6 than the drug Dexamethasone, the widely used corticosteroid medication to relieve inflammation (see the Supporting Information). More importantly, they did not show obvious cytotoxicity at the indicated concentrations compared to Dexamethasone. We further examined the effects of $\mathbf{6 g}$ on the levels of phosphorylation of NF-KB and IKB-a induced by LPS in RAW 246.7 cells. As expected, $\mathbf{6} \mathbf{g}$ could abrogate the phosphorylation of NF-KB and IкB-a, an NF-kB inhibitory protein, whose phosphorylation results in its degradation and promotes subsequent translocation of NF-KB into nucleus and transcription of inflammatory genes (Figure 2B).$^{70}$ The current preliminary pharmacological results indicated a promising prospect of $\mathbf{6 g}$ to be developed as a novel anti-inflammatory agent, with competitive potency and safety advantage.

Apart from the scope of these conversions and intriguing anti-inflammatory activities, we were also interested in the reaction mechanism. Two possible catalytic cycles are shown in Scheme 2. To gain insight into the proposed catalytic cycles and see which cycle is more favorable, we carried out DFT calculations to investigate the detailed mechanism. Although the similar mechanistic pathways have been proposed by others, ${ }^{42}$ the DFT calculations of Pd(II) shift are still uncovered to date.

Figure 3 shows the energy profiles calculated. Considering the sizes of ligand and substrate, we start with the complex $\mathbf{A}$ in which the $\mathrm{Pd}(0)$ metal center coordinates with the ligand $\mathrm{L}$ and the substrate aryl bromide [Figure 3(a)]. Oxidative addition (OA) followed by concerted metalation deprotonation (CMD) gives the key palladacycle intermediate IM4. The barrier for the $\mathrm{OA}$ is small while the barrier for the CMD process is $33.4 \mathrm{kcal} / \mathrm{mol}$.

From the key palladacycle intermediate IM4, two possible paths (consideration of the two cycles shown in Scheme 2) were calculated (Figure 3(b)). Path A involves alkyne-activation cyclization followed by migratory insertion (Cycle $A$ in Scheme 2), while Path $B$ engages protonation first and then alkyneactivation cyclization (Cycle B in Scheme 2). Clearly, Path A requires to pass through a very high-lying transition state $\left(\mathrm{TS}_{6-7 \mathrm{~A}}\right)$ for the migratory insertion. The high-lying $\mathbf{T S}_{6-7 \mathrm{~A}}$ structure is a result of the unfavorable migration step that involves weakening/breaking of the two strong $\mathrm{Pd}-\mathrm{C}$ bonds in the 5membered ring of IM6A. 
Figure 3(c) shows that when the migration insertion occurs on the carbene structure IM7B without a 5membered ring moiety, a very small barrier of $7.5 \mathrm{kcal} / \mathrm{mol}$ is calculated. After the migratory insertion, which is highly exergonic, $\beta$-hydride elimination occurs easily (almost barrierless), followed by reductive elimination and ligand (aryl bromide) coordination to regenerate the active species $\mathbf{A}$.

The calculation results suggest that Cycle $B$ is favorable. From Figure 3, we can also see that the CMD transition state structure $\mathbf{T S}_{\mathbf{2 - 3}}$ (Figure 3(a)) and the protonation transition state structure $\mathbf{T S}_{\mathbf{4 - 5 B}}$ (Path B in Figure 3(b)) show similar stability, although the latter lies slightly higher than the former. On the basis of the results, $\mathbf{T S}_{4-5 B}$ (Path B in Figure 3(b)) is the rate-determining transition state, and therefore, the overall reaction barrier is $34.1 \mathrm{kcal} / \mathrm{mol}$, corresponding to the energy difference between $\mathbf{I M 1}$ and $\mathbf{T S}_{\mathbf{4 - 5}}$. The calculated overall energy barrier is moderately high, which is understandable in view of the fact that the reaction temperature is $100^{\circ} \mathrm{C}$. In Figure 3, the series of transformation from IM2 to IM5B corresponds to a 1,4-Pd-shift.

Apart from all of the calculations mentioned above, we also calculated a pathway, which is closely related to Path A but starts from IM4B (instead of IM4) to react with 2a. The calculation results (Figure S1) indicate that this pathway is slightly favorable than Path A, but still less favorable than Path B.

\section{Conclusion}

In summary, we have developed two types of Pd-catalyzed intermolecular unactivated $\mathrm{Csp}^{3}-\mathrm{H}$ bond insertion reactions by choosing ene-yne-ketones and allenyl ketones as donor/donor carbene precursors, allowing for the construction of a diversity of alkenes substituted with furans and dihydrofurans. These two carbene cross couplings exhibit high efficiency and stereoselectivity, which can be applied to latestage cyclization/olefination of different therapeutic drugs. DFT mechanistic studies supported that a unique 1,4-Pd shift was involved in the catalytic cycle. Furthermore, alkenes substituted with furans as novel building blocks were successfully assembled via a short and modular biomimetic strategy into macrolactams, which showed significant anti-inflammatory activity with less cytotoxicity.

\section{Declarations}

\section{Data availability}

All relevant data are available in Supplementary Information, Supplementary Data and from the authors.

\section{Acknowledgements}

We gratefully acknowledge 100 talent program of Chinese Academy of Sciences, NSFC (21702217), "1000-Youth Talents Plan", Shanghai-Youth Talent, National Science \& Technology Major Project" Key New Drug Creation and Manufacturing Program" China (Number: 2018ZX09711002-006), and ShanghaiTechnology Innovation Action Plan (18JC1415300), and the Research Grants Council of Hong Kong (HKUST 16302418) for financial support of this research. 
These authors contributed equally: Jiping Hao, Xueying Guo, Shijun He.

Competing interests: The authors declare no competing interests.

\section{References}

1. Newman, D. J., Cragg, G. M., Macrocycles in Drug Discovery, Vol. 40 (Ed.: J. Levin), The Royal Society of Chemistry, Cambridge, 2015, pp. 1-36.

2. Ramsey, D. M., Kitson, R. R. A., Levin, J. I., Moody, C. J., McAlpine, S. R. Macrocycles in Drug Discovery, Vol. 40 (Ed.: J. Levin), The Royal Society of Chemistry, Cambridge, 2015, pp. 37-77.

3. Driggers, E. M., Hale, S. P., Lee, J., Terrett, N. K. The exploration of macrocycles for drug discovery-an underexploited structural class. Nat. Rev. Drug. Discov. 7, 608-624 (2008).

4. Mallinson, J., Collins I. Macrocycles in new drug discovery. Future Med. Chem. 4, 1409-1438 (2012).

5. Cummings, M. D., Sekharan, S. Structure-Based Macrocycle Design in Small-Molecule Drug Discovery and Simple Metrics To Identify Opportunities for Macrocyclization of Small-Molecule Ligands. J. Med. Chem. 62, 6843-6853 (2019).

6. Roethle, P. A., Trauner, D., The chemistry of marine furanocembranoids, pseudopteranes, gersolanes, and related natural products. Nat. Prod. Rep., 25, 298-317 (2008).

7. Li, Y., Pattenden, G., Perspectives on the structural and biosynthetic interrelationships between oxygenated furanocembranoids and their polycyclic congeners found in corals. Nat. Prod. Rep., 28, 1269-1310 (2011).

8. Mortensen, K.T., Osberger, T.J., King, T.A., Sore, H.F., Spring, D. R. Strategies for the Diversity-Oriented Synthesis of Macrocycles. Chem Rev 119, 10288-10317 (2019).

9. Reguera, L., Rivera, D. G. Multicomponent Reaction Toolbox for Peptide Macrocyclization and Stapling. Chem. Rev. 119, 9836-9860 (2019).

10. Marsault, E., Peterson, M. L. Macrocycles are great cycles: applications, opportunities, and challenges of synthetic macrocycles in drug discovery. J. Med. Chem. 54, 1961-2004 (2011).

11. Grossmann, A., Bartlett, S., Janecek, M., Hodgkinson, J. T., Spring, D. R. Diversity-oriented synthesis of drug-like macrocyclic scaffolds using an orthogonal organo- and metal catalysis strategy. Angew. Chem. Int. Ed. 53, 13093-13097 (2014).

12. Beckmann, H. S., et al. A strategy for the diversity-oriented synthesis of macrocyclic scaffolds using multidimensional coupling. Nat. Chem. 5, 861-867 (2013).

13. Frost, J. R., Scully, C. C., Yudin, A. K. Oxadiazole grafts in peptide macrocycles. Nat. Chem. 8, 11051111 (2016). 
14. McCarver, S. J., et al. Decarboxylative Peptide Macrocyclization through Photoredox Catalysis. Angew. Chem. Int. Ed. 56, 728-732 (2017).

15. Over, B., et al. Natural-product-derived fragments for fragment-based ligand discovery. Nat. Chem. 5, 21-28 (2013).

16. Hao, J., Xu, Y., Xu, Z., Zhang, Z., Yang, W. Pd-Catalyzed Three-Component Domino Reaction of Vinyl Benzoxazinanones for Regioselective and Stereoselective Synthesis of Allylic Sulfone-Containing Amino Acid Derivatives. Org. Lett. 20, 7888-7892 (2018).

17. Yang, Y., Yang, W. Divergent synthesis of N-heterocycles by Pd-catalyzed controllable cyclization of vinylethylene carbonates. Chem. Commun. 54, 12182-12185 (2018).

18. Deng, L., Kleij, A. W., Yang, W. Diversity-Orientated Stereoselective Synthesis through Pd-Catalyzed Switchable Decarboxylative C-N/C-S Bond Formation in Allylic Surrogates. Chem. Eur. J. 24, 1915619161 (2018).

19. Xu, Y., Chen L., Yang, Y., Zhang Z., Yang, W. Vinylethylene Carbonates as alpha,beta-Unsaturated Aldehyde Surrogates for Regioselective [3 + 3] Cycloaddition. Org. Lett. 21, 6674-6678 (2019).

20. Song, B., Xie, P., Li, Y., Hao, J., Wang, L., Chen, X., Xu, Z., Quan, H., Lou, L., Xia, Y., Houk, K. N., Yang, W. Pd-Catalyzed Decarboxylative Olefination: Stereoselective Synthesis of Polysubstituted Butadienes and Macrocyclic P-glycoprotein Inhibitors. J. Am. Chem. Soc. 142, 9982-9992 (2020).

21. Chen, L., Quan, H., Xu, Z., Wang, H., Xia, Y., Lou, L., Yang, W. A modular biomimetic strategy for the synthesis of macrolide P-glycoprotein inhibitors via Rh-catalyzed C-H activation. Nat. Commun., 11, 2151 (2020).

22. Xia, Y., Qiu, D., Wang, J. Transition-Metal-Catalyzed Cross-Couplings through Carbene Migratory Insertion. Chem. Rev. 117, 13810-13889 (2017).

23. Lyons, T. W., Sanford, M. S. Palladium-catalyzed ligand-directed C-H functionalization reactions. Chem. Rev. 110, 1147-1169 (2010).

24. He, J., Wasa, M., Chan, K. S. L., Shao, Q., Yu, J. Q. Palladium-Catalyzed Transformations of Alkyl C-H Bonds. Chem. Rev. 117, 8754-8786 (2017).

25. Chen, Z. K., Wang, B. J., Zhang, J. T., Yu, W. L., Liu, Z. X., Zhang, Y. H. Transition metal-catalyzed C-H bond functionalizations by the use of diverse directing groups. Org. Chem. Front. 2, 1107-1295 (2015).

26. Chan, W. W., Lo, S. F., Zhou, Z., Yu, W. Y. Rh-catalyzed intermolecular carbenoid functionalization of aromatic C-H bonds by alpha-diazomalonates. J. Am. Chem. Soc. 134, 13565-13568 (2012).

27. Zhao, D., Kim, J. H., Stegemann, L., Strassert, C. A., Glorius F. Cobalt(III)-catalyzed directed C-H coupling with diazo compounds: straightforward access towards extended pi-systems. Angew. Chem. Int. Ed. 54, 4508-4511 (2015).

28. Hyster, T. K., Ruhl, K. E., Rovis, T. A coupling of benzamides and donor/acceptor diazo compounds to form gamma-lactams via Rh(III)-catalyzed C-H activation. J. Am. Chem. Soc. 135, 5364-5367 (2013). 
29. Yu, S., Liu, S., Lan, Y., Wan, B., Li, X. Rhodium-catalyzed C-H activation of phenacyl ammonium salts assisted by an oxidizing C-N bond: a combination of experimental and theoretical studies. J. Am. Chem. Soc. 137, 1623-1631 (2015).

30. Xie, H., Ye, Z., Ke, Z., Lan, J., Jiang, H., Zeng, W. Rh(iii)-catalyzed regioselective intermolecular Nmethylene Csp(3)-H bond carbenoid insertion. Chem. Sci. 9, 985-989 (2018)

31. Zhou, B., et al. Redox-neutral rhodium-catalyzed C-H functionalization of arylamine N-oxides with diazo compounds: primary $\mathrm{C}(\mathrm{sp}(3))-\mathrm{H} / \mathrm{C}(\mathrm{sp}(2))-\mathrm{H}$ activation and oxygen-atom transfer. Angew. Chem. Int. Ed. 54, 12121-12126 (2015).

32. Davies, H. M. L., Venkataramani, C. Catalytic enantioselective synthesis of beta(2)-amino acids. Angew. Chem. Int. Ed. 41, 2197-2199 (2002).

33. Guptill, D. M., Davies, H. M. 2,2,2-Trichloroethyl aryldiazoacetates as robust reagents for the enantioselective C-H functionalization of methyl ethers. J. Am. Chem. Soc. 136, 17718-17721 (2014).

34. Davies, H. M., Morton, D. Guiding principles for site selective and stereoselective intermolecular C-H functionalization by donor/acceptor rhodium carbenes. Chem. Soc. Rev. 40, 1857-1869 (2011).

35. Solé, D., Mariani, F., Bennasar, M.-L., Fernández, I., Palladium-catalyzed intramolecular carbene insertion into C(sp3)-H bonds. Angew. Chem. Int. Ed. 55, 6467-6470 (2016).

36. Davies, H. M. L., Liao, K. B. Dirhodium tetracarboxylates as catalysts for selective intermolecular C-H functionalization. Nat. Rev. Chem. 3, 347-360 (2019).

37. Davies, H. M. L., Hansen, T. Asymmetric intermolecular carbenoid C-H insertions catalyzed by rhodium(II) (S)-N-(p-dodecylphenyl)sulfonylprolinate. J. Am. Chem. Soc. 119, 9075-9076 (1997).

38. Liao, K., Negretti, S., Musaev, D. G., Bacsa J., Davies, H. M. Site-selective and stereoselective functionalization of unactivated C-H bonds. Nature 533, 230-234 (2016).

39. Liao, K., Pickel, T. C., Boyarskikh, V., Bacsa, J., Musaev, D. G., Davies, H. M. L. Site-selective and stereoselective functionalization of non-activated tertiary C-H bonds. Nature 551, 609-613 (2017).

40. Liao, K., et al. Design of catalysts for site-selective and enantioselective functionalization of nonactivated primary C-H bonds. Nat. Chem. 10, 1048-1055 (2018).

41. Pena-Lopez, M., Beller, M. Functionalization of Unactivated C(sp(3))-H Bonds Using Metal-Carbene Insertion Reactions. Angew. Chem. Int. Ed. 56, 46-48 (2017).

42. Gutierrez-Bonet, A., Julia-Hernandez, F., Luis, B., Martin, R. Pd-Catalyzed C(sp(3))-H Functionalization/Carbenoid Insertion: All-Carbon Quaternary Centers via Multiple C-C Bond Formation. J. Am. Chem. Soc.138, 6384-6387 (2016).

43. Jia, M., Ma, S. New Approaches to the Synthesis of Metal Carbenes. Angew. Chem. Int. Ed. 55, 91349166 (2016).

44. Zhu, D., Ma, J., Luo, K., Fu, H., Zhang, L., Zhu, S. Enantioselective Intramolecular C-H Insertion of Donor and Donor/Donor Carbenes by a Nondiazo Approach. Angew. Chem. Int. Ed. 55, 8452-8456 (2016). 
45. Ma, J., Zhang, L., Zhu, S. Enynal/Enynone: A Safe and Practical Carbenoid Precursor. Curr. Org. Chem., 20, 102-118 (2015).

46. Qian, D., Zhang, J. Gold-catalyzed cyclopropanation reactions using a carbenoid precursor toolbox. Chem. Soc. Rev. 44, 677-698 (2015).

47. Xia, Y., et al. Oxidative cross-coupling of allenyl ketones and organoboronic acids: expeditious synthesis of highly substituted furans. Angew. Chem. Int. Ed. 53, 3917-3921 (2014).

48. Hashmi, A. S., Schwarz, L., Choi, J. H., Frost, T. M. A New Gold-Catalyzed C-C Bond Formation. Angew. Chem. Int. Ed. 39, 2285-2288 (2000).

49. Sromek, A. W., Rubina, M., Gevorgyan, V. 1,2-Halogen migration in haloallenyl ketones: regiodivergent synthesis of halofurans. J. Am. Chem. Soc. 127, 10500-10501 (2005).

50. Xia, Y., et al. Palladium-catalyzed carbene migratory insertion using conjugated ene-yne-ketones as carbene precursors. J. Am. Chem. Soc. 135, 13502-13511 (2013).

51. Zhu, D., Chen, L., Fan, H., Yao, Q., Zhu, S. Recent progress on donor and donor-donor carbenes Chem. Soc. Rev., 49, 908-950 (2020).

52. Rahim, A., Feng, J., Gu, Z. H. 1,4-Migration of Transition Metals in Organic Synthesis. Chinese Journal of Chemistry 37, 929-945 (2019).

53. Ma, S., Gu, Z. 1,4-migration of rhodium and palladium in catalytic organometallic reactions. Angew. Chem. Int. Ed. 44, 7512-7517 (2005).

54. Piou, T., Bunescu, A., Wang, Q., Neuville, L., Zhu, J. Palladium-catalyzed through-space C(sp(3))-H and $\mathrm{C}(\mathrm{sp}(2))-\mathrm{H}$ bond activation by 1,4-palladium migration: efficient synthesis of [3,4]-fused oxindoles. Angew. Chem. Int. Ed. 52, 12385-12389 (2013).

55. Barder, T. E., Walker, S. D., Martinelli, J. R., Buchwald, S. L. Catalysts for Suzuki-Miyaura coupling processes: scope and studies of the effect of ligand structure. J. Am. Chem. Soc. 127, 4685-4696 (2005).

56. Hu, T. J., Zhang, G., Chen, Y. H., Feng, C. G., Lin, G. Q. Borylation of Olefin C-H Bond via Aryl to Vinyl Palladium 1,4-Migration. J. Am. Chem. Soc. 138, 2897-2900 (2016).

57. Campo, M. A., Larock, R. C. Novel 1,4-palladium migration in organopalladium intermediates derived from o-iodobiaryls. J. Am. Chem. Soc. 124, 14326-14327 (2002).

58. Wang, Q. N., Chen, R. J., Lou, J., Zhang, D. H., Zhou, Y. G., Yu, Z. K. Highly Regioselective C-H Alkylation of Alkenes Through an Aryl to Vinyl 1,4-Palladium Migration/C-C Cleavage Cascade. ACS Catal. 9, 11669-11675 (2019).

59. Rocaboy, R., Anastasiou, I., Baudoin, O. Redox-Neutral Coupling between Two C(sp(3))-H Bonds Enabled by 1,4-Palladium Shift for the Synthesis of Fused Heterocycles. Angew. Chem. Int. Ed. 58, 14625-14628 (2019).

60. Wang, M., Zhang, X., Zhuang, Y. X., Xu, Y. H., Loh, T. P. Pd-catalyzed intramolecular C-N bond cleavage, 1,4-migration, sp3 $\mathrm{C}$-H activation, and Heck reaction: four controllable diverse pathways 
depending on the judicious choice of the base and ligand. J. Am. Chem. Soc. 137, 1341-1347 (2015).

61. Yu, Y., Chakraborty, P., Song, J., Zhu, L., Li, C., Huang, X. Easy access to medium-sized lactones through metal carbene migratory insertion enabled 1,4-palladium shift Nat. Commun. 2020, 11, 461.

62. Burnet, M., Guse, J., Gutke, H., Guillot, L., Laufer, S., Hahn, U., Seed, M. P., Vallejo, E., Eggers, M., Mckenzie, D., Albrecht, W., Parnham, M. J. Macrocycles in Drug Discovery, Vol. 40 (Ed.: J. Levin), The Royal Society of Chemistry, Cambridge, 2015, pp. 206-234.

63. Baudoin, O. Ring Construction by Palladium(0)-Catalyzed C(sp(3))-H Activation. Acc. Chem. Res. 50, 1114-1123 (2017).

64. Lu, A., Ji, X., Zhou, B., Wu, Z., Zhang, Y. Palladium-Catalyzed C-H Silylation through Palladacycles Generated from Aryl Halides. Angew. Chem. Int. Ed. 57, 3233-3237 (2018).

65. Tan, B., Bai, L., Ding, P., Liu, J., Wang, Y., Luan, X. Palladium-Catalyzed Intermolecular [4 + 1] Spiroannulation by C(sp(3))-H Activation and Naphthol Dearomatization. Angew. Chem. Int. Ed. 58, 1474-1478 (2019).

66. Sun, X., Wu, Z., Qi, W., Ji, X., Cheng, C., Zhang, Y. Synthesis of Indolines by Palladium-Catalyzed Intermolecular Amination of Unactivated C(sp(3))-H Bonds. Org. Lett. 21, 6508-6512 (2019).

67. Sarkar, F. H., Li, Y., Wang, Z., Kong, D. NF-kappaB signaling pathway and its therapeutic implications in human diseases. Int. Rev. Immunol. 27, 293-319 (2008).

68. Baker, R. G., Hayden, M. S., Ghosh, S. NF-kappaB, inflammation, and metabolic disease. Cell Metab. 13, 11-22 (2011).

69. Zhao, J. X., et al. Structural Elucidation and Bioinspired Total Syntheses of Ascorbylated Diterpenoid Hongkonoids A-D. J. Am. Chem. Soc. 140, 2485-2492 (2018).

70. Karin, M., Greten, F. R. NF-kappaB: linking inflammation and immunity to cancer development and progression. Nat. Rev. Immunol. 5, 749-759 (2005).

\section{Tables And Schemes}

Due to technical limitations, full-text HTML conversion of the tables and schemes could not be completed. However, they can be downloaded and accessed in the Supplementary Files.

\section{Figures}


A Marine Furanocembranoids and natural or pseudo-natural scaffoids

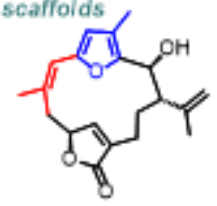

Bipinnazก J<smiles>C=C(C)C(Cc1oc(/C=C\CC2C=C(CC(=O)OC)C(=O)O2)cc1C(=O)O)OC</smiles>

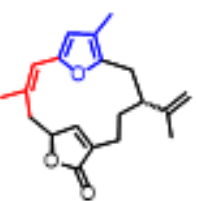

Rubifolide

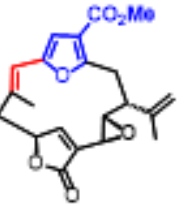

Acerolide

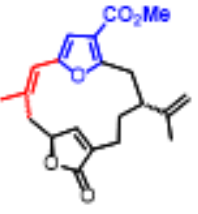

Z-Deoxypukainde E-Deoxypukalide

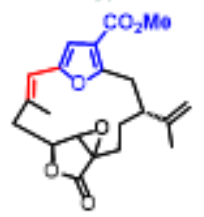

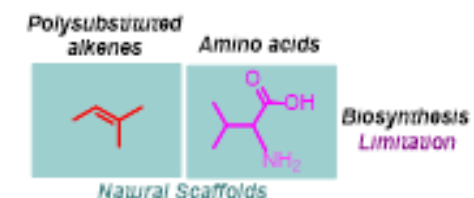

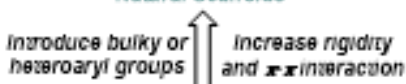

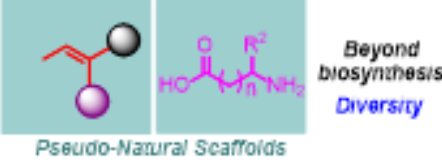

B Marine Furanocembranoids-inspired modularization strategy for synthesis of polysubstituted alkenes furanembedded macrolactams.
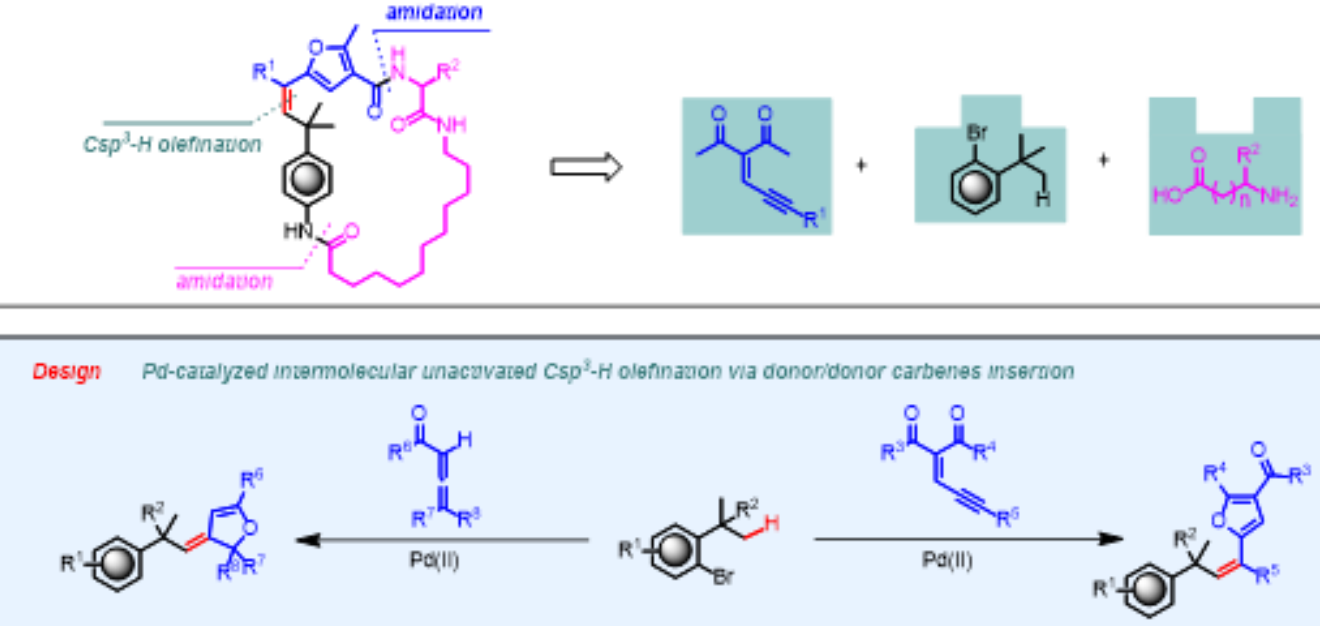

Feazures:

口 High stereoselectivity Q First Csp ${ }^{3}-\mathrm{H}$ insertion reactions of donoridonor carbenes

口Polysubstituted alkenes with heterocycllcs a 1,4-Pd shift DFT calculation

The staze of art of carbene inservion unvo Csp ${ }^{3}-\mathrm{H}$ bond:

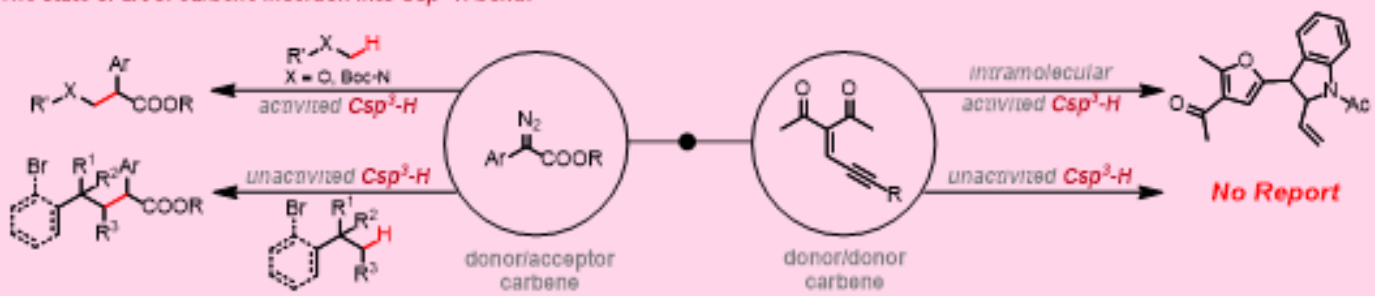

Challenges: $\quad$ Q Low nuclephllic reactivity of $\mathrm{Cap}^{3} \cdot \mathrm{H}$ bonds

Q Weak electrophillc capacity of donoridonor carbene

口 Compoting with oxidation and dimerization

\section{Figure 1}

A modular biomimetic strategy for the synthesis of natural furanocembranoids-like via Pd-catalyzed unactivated $\mathrm{Csp} 3-\mathrm{H}$ insertion reaction of donor/donor carbenes 

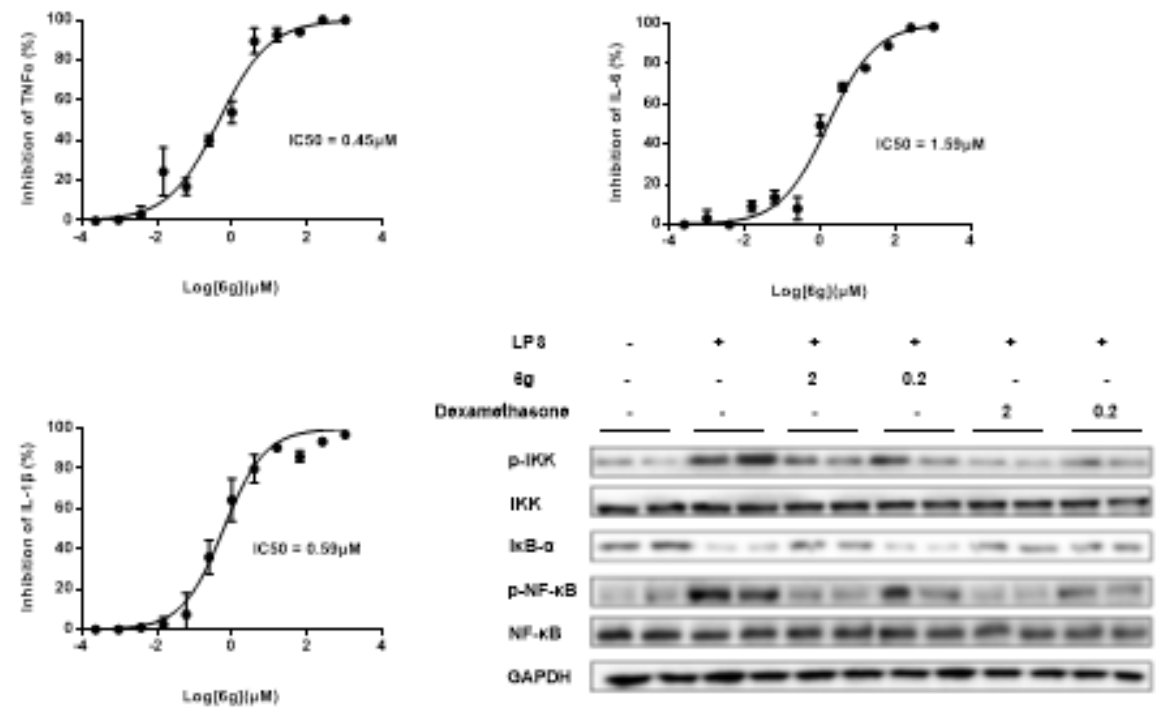

\section{Figure 2}

Compound $6 \mathrm{~g}$ suppressed the LPS-induced inflammatory responses in RAW264.7 macrophages. IC50: the concentration of the compound needed to inhibit inflammatory mediators by $50 \%$ relative to the control value. Effect of $6 \mathrm{~g}$ on LPS-induced TNF- $a$, IL- 6 and IL-1 $\beta$. Cells were treated with LPS alone (5 $\mu \mathrm{g} / \mathrm{mL}$ ) or with indicated 


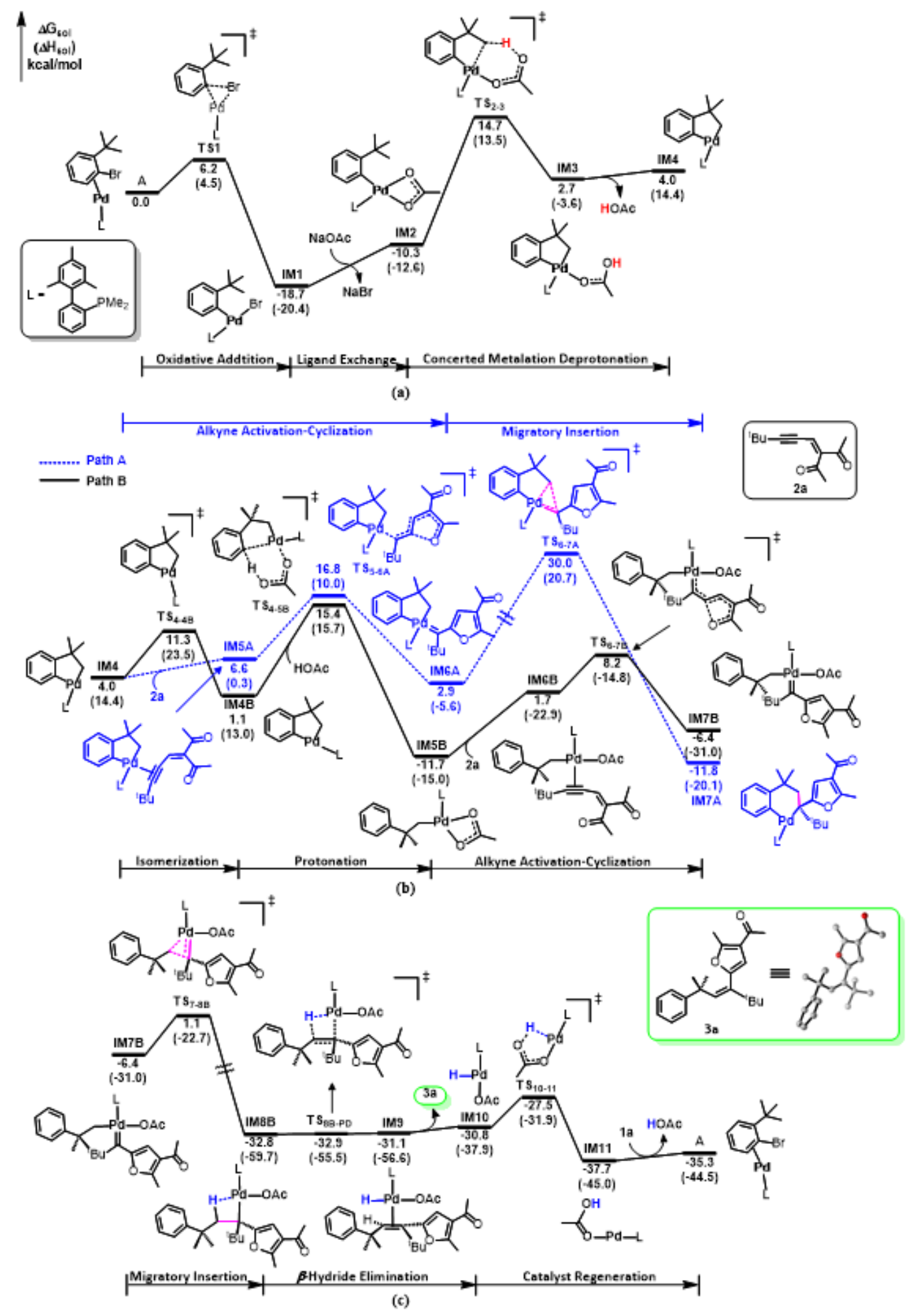

\section{Figure 3}

Energy profiles calculated on the basis of the mechanistic cycles shown in Scheme 2. (a) Oxidative addition followed by CMD leading to the key palladacycle intermediate IM4, (b) two different paths starting from IM4, and (c) catalyst regeneration for the favorable path (Cycle B). The solvation-corrected relative free energies and electronic energies (in parentheses) are given in $\mathrm{kcal} / \mathrm{mol}$. 


\section{Supplementary Files}

This is a list of supplementary files associated with this preprint. Click to download.

- supplement1.docx

- supplement2.docx

- supplement3.cif

- supplement4.cif 